\title{
Educational Environment and the Application of IT in Pharmacy Education
}

\author{
Mariana Drachuk* \\ Lecturer of the Department of Biophysics, Danylo Halytskyi Lviv National Medical University, Lviv, Ukraine \\ *Corresponding author: maryshka0503@gmail.com
}

Received September 16, 2014; Revised October 23, 2014; Accepted December 14, 2014

\begin{abstract}
The article deals with the problem of educational environment and application of information technology (IT) in Pharmacy education. It has been investigated the formation of professional knowledge using information technology in Pharmacology and its application as an educational tool in the professional training system aimed at teaching future pharmacists and preparing students to use IT in their professional activity. For this purpose the training course "Information Technology in Pharmacy" has been developed and included into the curriculum. The development and implementation of this course into the curriculum proved to be efficient and successful. The pedagogical conditions of the application of IT in professional training of pharmacists have been theoretically grounded. Computer science in the sphere of health care or medical computer science is a new branch that requires appropriate personnel training. Thus, it is necessary to develop training programs to ensure efficient training of specialists and implement information technology in medical science and health care.
\end{abstract}

Keywords: information technology, pedagogical conditions, professional training, pharmacy education, training of highly-qualified specialists, competency approach

Cite This Article: Mariana Drachuk, "Educational Environment and the Application of IT in Pharmacy Education." American Journal of Educational Research, vol. 2, no. 12B (2014): 56-60. doi: 10.12691/education2-12B-11.

\section{Introduction}

According to the concept of reforming the medical education in Ukraine and bringing it into line with the requirements of the Bologna Declaration, the main purpose of practical professional training is "consolidating the theoretical knowledge through practical training, mastering the modern methods and approaches of providing medical care, learning the new technologies, forming and developing professional skills and competences in order to make independent decisions, developing the need for systematic upgrading of knowledge and long life learning and finally, psychological adaptation to the specific forms of professional activity." [1].

In modern conditions the quality of education is associated with the objectives of the Bologna Process. The main goal of education in such conditions is the preparation of well-qualified professionals who will be efficient in performing their work and competitive in the labor market. In order to achieve this goal students are to gain the high-level knowledge. Moreover, the new technologies which are adapted to the specific working environment should be developed and included in the education process on its various stages. Also, the new technology should become a guide for new solutions [3].
A special attention is paid to the training of new professionals - clinical pharmacists who are supposed to work together with a doctor and carry out ward rounds, work in a hospital, conduct therapeutic practice, be acknowledged of the state medical policy, regulation of medication provision and reserves, the concept of essential medicines and their rational use. The main objective of a clinical pharmacist is to improve the medical care of patients as well as to create appropriate conditions for safe and rational use of drugs. The main aspects of improving the training of pharmacists are as follows: fundamentalization of education, gradual transition to the problematic investigative approaches of studying, continual and steady functioning of the cycle «educational institution - student - employer», enhancing the role of scientific and pedagogical forecasting of all the components of the teaching and educational system as well as applying information technologies in the educational process.

In modern pedagogical science the great attention is paid to research of the problems of professional training of future pharmacists (I. Boichuk, O. Zalyubovska, L. Kaidalova, O. Velchynska, V. Chornykh, V. Tolochko and others), application of information technology in professional training (A. Kolomiyets, V. Izvozchykov, V. Laptyev, M. Potyomkin, E. Tumalyev and others) and issues of application of IT in Pharmacy and pharmaceutical education (V. Mozul, Ye. Knysh, T. Raikova, T. Zarichna, A. Mazulin, O. Surikov and others). 
However, the educational environment and pedagogical conditions of the application of IT in the professional training of pharmacists have not been researched sufficiently and that was the reason for choosing this subject to be discussed in the paper.

\section{Research Methodology}

The objective of the article is to introduce the theoretical grounds for educational environment of the application of information technology in the professional training of pharmacists.

Nowadays there is a demand for the new approach to criteria development for the effective use of information resources in the system of professional education. According to this approach the system of information requirements appropriate to the professional education system in terms of structure, composition and context should be developed. Also, there is a need for the mechanisms of effective application of information resources within the system of professional education which can be based on the analysis of efficiency of information resources used in various departments of the professional education system operating in the mode of innovations.

The emphasis is put on training of new professionals clinical pharmacists. The main objective of a clinical pharmacist is to improve the medical care of patients as well as to create appropriate conditions for safe and rational use of drugs. The main aspects of Pharmacy education are fundamentalization of education, gradual transition to the problematic and investigative approaches of training, continual and steady functioning of the cycle «educational institution - student - employer», enhancing of the role of scientific and pedagogical forecasting all the components of the teaching and educational system as well as applying information technologies in the educational process.

\section{Discussion and Results}

Based on the competency approach a number of features of the professional information competence of pharmacists could be outlined. They include computer skills sufficient to the level of professional education, the necessary minimum of command of foreign languages, the high level of communicative culture, having theoretical basis and experience in organizing the communicative information interaction, possessing the skills of receiving, selecting, reproducing, transforming the ways of presenting information, transmitting and integrating information, combining professional knowledge and computer skills in the due course of professional activity as well as the ability to foresee the results of their professional and information activity and the formation of professional experience in modern information environment both national and international.

The system of values according to which a man acts is his personal choice. However, such subject as Computer Science and Information Technology helps a person to perceive the surrounding reality. [4] This determines the need to use the cultural approach to the application of information technology in the professional training of pharmacists. Taking into account the above stated, the factors necessary to the process of the formation of information culture of pharmacists during their professional education have been identified. They are as follows: objective or external factors which are characterized by the influence of the whole society; objectively subjective or complex factors such as a type of educational institution, social requirements to the content and level of education of the future pharmacists, demands of a labor market, etc.; subjective or internal factors like the real space of teaching-learning interaction between teachers and students in a particular institution.

There are four complementary aspects of the systematic approach to training of students specializing in Pharmacy: interdisciplinary, a balanced combination of professional and computer training, systematic study of subjects of the information and computer sciences cycle as well as systematic professional training in general.

Accordingly, the competency, cultural and systematic approaches constitute the methodological basis of the application of information technology in Pharmacy education.

On this ground the pedagogical conditions of the application of IT in training of pharmacists could be outlined. The first pedagogical condition provides the formation of knowledge on how to use information technology in Pharmacy.

With the development of medicine and pharmacy certain criteria for the effectiveness of treatment were introduced. For a long time laboratory parameters have played a role of such criteria. The important question is to determine the list of reference drugs and to create and publish directories establishing a therapeutic equivalence in Ukraine [7].

Under current conditions modeling helps to determine directions of the development of pharmacy computerization and to improve the current versions of software by introducing expert systems.

In order to be professional a clinical pharmacist should use modern information technology. A special attention is paid to the application of modern information systems which allow pharmaceutical companies and medical institutions to maintain contact with each other on the regular basis. Only those manufacturers that apply new technology in the analysis of clinical implementation of new drugs can achieve maximum success.

Computer science in the sphere of health care or medical computer science is a new area that requires appropriate personnel training. For this purpose it is necessary to develop training programs to ensure efficient training of specialists and implement information technology in medical science and health care.

In their professional activity pharmacists apply information technology on the following stages: creation of a drug; pharmacological screening (there are programs which are used to foresee the biological effects of a synthesized substance); marketing research of the pharmaceutical market; registration and accounting of medicines and the information and reference service of pharmaceutical companies and pharmacies.

In modern conditions instrumental, hardware, software and pharmaceutical support of advanced medical technologies is the only process that can integrate the 
intellectual potential of specialists in various fields of science and technology and is characterized by the formulation of a field of science with an integrated structure - medical and technical science.

There are such programs that apply a new classification of drugs and by using certain mathematical models can determine the possible success or failure of a new drug and examine the therapeutic possibilities of drugs which have the same pharmaceutical properties as well as further horizons of the development of drugs.

In the process of synthesis of a new drug there is a constant need for information on pharmacological activity of different substances and drugs. There is a database (DB) to service such requests. Moreover, there is a specially designed coding system which describes the effects of a particular drug as well as their mechanisms, allowing to select an effective drug taking into consideration similar properties. The ethical aspects associated with drugs seem to be equally significant - ranging from research and production to support of marketing departments and pharmaceutical companies.

The main directions of the development of modern information technology in pharmaceutical industry include the automation of document circulation; specialists' adaptation to the flow of information; the ability to select, evaluate and use information; the ability to self-education and a need for upgrading the knowledge and regular advanced training; competence in IT tools and practical skills on how to use them; communication skills and the ability to work in a team, etc.

The second pedagogical condition involves the application of information technology as a means of professional training of pharmacists.

At present there is a demand for a new approach to the development of criteria of the effective use of information resources. In the framework of this new approach a system of information requirements appropriate to the structure, content and nature of the system of vocational education will be created. Also, there is a need for the mechanisms that can ensure the effective use of information resources based on the analysis of effective application of information resources in terms of vocational education [9].

The main attention in addressing problems related to teaching of computer technology in vocational training should be paid to the development of content, methodological support and organization of information and technical training in vocational educational institutions. Based on the knowledge of new information technologies gained at school, the main areas of information and technical training in the system of professional education are developed. The main focus is put on the knowledge and skills in processing the digital and textual information based on the integrated information and communicative networks, automated control and regulation technology during the process of production, conducting measurements, during the operation of equipment containing the microprocessor technology as well as automated construction and design systems [5]. This creates prerequisites for designing the integrative course on computer technology with the elements of professional knowledge that allows to combine professional knowledge with the fundamentals of computer technology and later apply them jointly in professional activity.
The role of a computer in education is identified by the type of a training computer program. Such programs have great possibilities to implement the learning tasks. These systems perform reflexive management of the educational process. Typically, they take into account not only the correct answers but also a way of solving the problem.

Describing the various training programs, it is necessary to take into consideration what students' functions are activated. The following types of functions can be outlined: creating positive motivation, explanation, command and fixation of learning skills and knowledge; organization and monitoring of the students' performance; transfer of the routine component of learning process to a computer; development of training programs appropriate to different stages of the learning process as well as to individual peculiarities of students [2].

The analysis of various aspects of the problem of information support of Pharmacy education defines the relevance of the use of instruments for the creation of information environment adequate to current information conditions.

The above suggests that the use of information technology in professional training of pharmacists includes the following issues:

- Considering the specifics of professional activity of pharmacists;

- Maintenance and computer support of the entire educational process;

- Development and implementation of the course "Information Technologies in Pharmacy";

- Orientation on the current state and prognostic trends in the development of pharmaceutical industry in the context of the application of computer technology;

- Organization of students' independent work using information technologies;

- Adjusting the general objectives of computer use in education to the specifics of professional training;

- Information culture as a component of the professional culture of a specialist;

- Using multimedia technology in the educational process.

The third condition involves training students by offering them the course "Information Technologies in Pharmacy" so that they could efficiently apply information technology in their professional activity.

Modernization of educational content within the computer science education provides for implementation of the optimal content of information and computer training of a specialist. Nowadays the fundamental information knowledge is being shaped on a qualitatively new level having a general social, technical and technological importance for each of groups of specialties which are specific in terms of information. Educational standards for computer science education cannot be considered as such developed once and for all. We believe that this problem can be solved in the framework of the formation of the theory and technology of dynamically updated educational standards for a particular education field. One of the most important issues is the development of ways and methods of the controlled independent work of students. The significance of the increasing focus on self-education that has been theoretically grounded and experimentally tested is obvious. This can be explained by 
the fact that according to the educational standards a significant amount of educational hours is allocated to self- education, the methodological support and control of which are not regulated [9]. The priority of the creative approach to mastering and performing the information activity focused on the dynamically changing information needs of the individual and society seems to be a specific issue.

Integration of information technologies into the work of pharmaceutical companies requires the introduction of new forms and tools in the educational courses for pharmacists-interns and pharmacists-trainees. This would contribute to the intensification of work and development of operational thinking while performing professional duties.

Availability of digital communications creates conditions for the formation of a common information educational environment that ensures the implementation of the principles of democratic education and open access to information and knowledge. The transmission speed and volume of information received by society in the process of its development are increasing in exponential progression. This is especially true for medical and pharmaceutical information [6]. Correspondingly, the requirements to education content of a modern specialist are also changing. The modern education system should not only give a certain amount of knowledge, skills and abilities but also technologies for self-education of specialists, the system of professional knowledge and practical skills based on information and communication technologies.

In order to become a professional pharmacist it is necessary to gain the knowledge and practical skills in the use of information technology. Production of pharmaceuticals is based on new technology that requires the knowledge of modern computer programs and the proper use of databases available on the Internet. The course "The Basics of Information Technology" is studied by the first and second-year students. The ultimate objectives of this subject is as follows:

- to identify the possibilities of applying information technology and means of computer technology in medicine and pharmacy;

- to demonstrate the skills in working with a personal computer and the ability to search for biomedical and pharmaceutical information using information technology;

- to explain the principles of formalization and algorithmization of medical and pharmaceutical tasks, the principle of modeling in biology, medicine and pharmacy;

- to use the methods of processing biomedical and pharmaceutical information;

- to apply the tools of information technology for solving problems in pharmacy;

- to use the special professional software;

- to apply modern computer communication technologies;

The fourth-year students study the course "Computer Technology in Pharmacy" the main objectives of which are as follows:

- to learn the approaches and principles of search for specialized biomedical as well as chemical and pharmaceutical information;
- to familiarize with the main packages of modern computer programs used in pharmacy;

- to learn to use databases of specialized information available on the Internet or electronic storage media;

- to be able to search for specialized information;

- to familiarize with the innovative technologies in pharmacy, particularly in pharmaceutical and medical chemistry and proper software;

- to learn the basic techniques of obtaining structural information, creating of databases, virtual screening (molecular modeling, docking, QSAR-analysis, optimization of the structure of leader compounds, combinatorial chemistry, high-performance screening, etc.) as well as appropriate software for their implementation;

- to familiarize with the latest pharmaceuticals and leader structures that have been designed using computer technology;

- to explain the principles of formalization and algorithmization of the medical and pharmaceutical problems, the principles of modeling in biology, medicine and pharmacy;

- to use the methods of processing medical and biological information as well as pharmaceutical information;

- to apply the tools of information technology for solving problems in pharmacy;

- to use the special professional software;

- to apply modern computer communication technologies.

The topics of the course "The Basics of Information Technology" are based on the curriculum of a school course of Computer Science. In this subject the function of control and self-management of the process of learning is clearly distinctive whereas the teacher's task is to create learning situations and guide the activities of students.

In this context it is useful to outline the common requirements for the development of the course "Information Technology in Pharmacy":

- formation of the professional knowledge and skills associated with the use of computer technology;

- formation of students' skills to work independently with computer technology;

- practical orientation of computer training aimed at the development of professional skills of students;

- successive and perspective development of the content, organization forms, methods and means of integrative learning of computer technology in professional training;

- ensuring the professional orientation of computer technology learning depending on the specifics of profession;

- differentiation of the course by the level of complexity of teaching material;

- variability of computer technology learning in vocational education depending on the level of proficiency.

The course "Computer Technology in Pharmacy" is studied as a profile subject and its content is coordinated with the objectives of other major subjects which determine the education profile of a particular profession. The study of information technology as a means of addressing professional tasks and using information systems are of the priority importance [8]. 


\section{Conclusion}

From this standpoint, training of highly-qualified specialists depends on the continuity of education and the balanced combination of fundamental and professional education. Therefore, the following grounded pedagogical conditions of the application of information technology in pharmacy could be outlined: the formation of knowledge on the use of information technology in Pharmacy, application of information technology as a means of the system of professional training of pharmacists, training students on how to apply information technology in their professional activity and training students by offering them the course "Information Technologies in Pharmacy" so that they could efficiently apply information technology in their professional activity.

\section{Recommendation}

The further areas of research include the development of the appropriate forms and methods of the application of information technology in professional education of pharmacists.

\section{References}

[1] The concept of reforming the system of physicians training in Ukraine and its adaptation to the requirements of the Bologna Declaration: Project, The Center of testing of the professional competence, 2001

[2] Novik, I.B. On the modeling of complex systems, Moscow, 1965, 126.

[3] Osin, A.V. Multimedia in education: the context of information, Moscow, 2004, 320.

[4] Tumaleva,E.A. "Formation of professional competence in information-technology training in the field of education," Proceedings of the A.I. Herzen Russian State Pedagogical University, vol. 4 (9), 139-148.

[5] Sobko, R.M. Didactic features of integration teaching of computer technology in the training of electricians, Kyiv, 2002, 222.

[6] Mintser, O. "The role of information technology in the stages of reforming medical education," Implementation of credit-modular system of educational process in the HM (pharmaceutical) EI in Ukraine: the results of problems and perspectives: materials of Educational and scientific conference with international participation, 20-21 May 2010.

[7] Surikov, O.O. "Scientific and methodological approaches to the development of computer information systems in the pharmacy program," VII National Congress of Pharmacists Ukraine, 15-17 September 2010, Kharkiv, 2010, 541.

[8] Lesyk, B.R. Integrated curriculum of the course "Computer Technologies in Pharmacy" for the fifth-year students of the Faculty of Pharmacy, Davylo Halytskyi Lviv State University, 2002.

[9] Kirilova, H.I. Informational training in secondary Technical School in accordance with the requirements of the labor market (research priorities), Educational Portal of Khanty-Mansiysk Autonomous District, http://www.eduhmao.ru. 